\title{
Universiteit
}

Leiden

The Netherlands

\section{Geometry and topological order in the Luttinger liquid state}

Kruis, H.V.; McCulloch, I.P.; Nussinov, Z.; Zaanen, J.

\section{Citation}

Kruis, H. V., McCulloch, I. P., Nussinov, Z., \& Zaanen, J. (2004). Geometry and topological order in the Luttinger liquid state. Retrieved from https://hdl.handle.net/1887/5133

Version: $\quad$ Not Applicable (or Unknown)

License: $\quad$ Leiden University Non-exclusive license

Downloaded from: $\quad$ https://hdl.handle.net/1887/5133

Note: To cite this publication please use the final published version (if applicable). 
Europhys. Lett., 65 (4), pp. 512-518 (2004)

DOI: $10.1209 / \mathrm{epl} / \mathrm{i} 2003-10114-3$

\title{
Geometry and topological order in the Luttinger liquid state
}

\author{
H. V. Kruis, I. P. McCulloch, Z. Nussinov and J. ZaAnen \\ Instituut-Lorentz for Theoretical Physics, Leiden University \\ P.O.B. 9506, 2300 RA Leiden, The Netherlands
}

(received 6 May 2003; accepted 5 December 2003)

PACS. 71.27. $+\mathrm{a}-$ Strongly correlated electron systems; heavy fermions.

PACS. 75.10.-b - General theory and models of magnetic ordering.

PACS. 71.10.Fd - Lattice fermion models (Hubbard model, etc.).

\begin{abstract}
We demonstrate the existence of a form of topological order in the Luttinger liquid state, which appears crucial to the notion of spin-charge separation. This order, which is closely related to the hidden order characterizing the Haldane spin chains, appears to be incompletely specified in the Tomonaga-Luttinger picture from bosonization. Thus, we use numerical calculations to complete the picture.
\end{abstract}

Systems composed of an infinite number of interacting quantum particles can be characterized by long-wavelength excitations carrying quantum numbers unrelated to those of the constituents. A classic example is the phenomenon of spin-charge separation which is well established in one-dimensional electron systems [1]. The elementary excitations can be viewed as pieces of the original electron, carrying separately the charge and the spin. This notion first emerged when it was demonstrated that systems of relativistic fermions in $1+1 \mathrm{D}$ can be represented exactly under a variety of circumstances in terms of Bose fields, carrying separately the spin and the charge degrees of freedom. Although this bosonization correctly describes asymptotic properties of local correlation functions, a possible loophole is that the lattice problem is characterized by a non-critical topological order. Here we demonstrate that at least the Hubbard model in $1+1 \mathrm{D}$ carries such an order, and we believe that the Hubbard model is in this regard fully representative for all non-relativistic electron systems. This topological order is a close relative of the hidden order discovered by den Nijs and Rommelse in the Haldane $(S=1)$ spin chains [2]. By virtue of the mapping onto a $t-J^{z}$ model with an additional $U(1)$ (charge) breaking superconducting term, Batista and Ortiz demonstrated that this order can exist in one-dimensional fermion systems [3]. Here we show that this order is crucial to the notion of spin-charge separation. Starting from a $Z_{2}$ sublattice parity description, we present arguments for the existence of a state of matter described by an effective $Z_{2}$ gauge theory.

This hidden order has to do with the fact that the charge carriers ("holons") are at the same time Ising "domain walls" (kinks) in the spin system, a property shared with stripes in two-dimensional systems [4]. Resting on the geometrical definition of spin-charge separation as deduced from the Bethe-Ansatz [5] solution of the Hubbard model at infinite $U$, obtained (c) EDP Sciences 


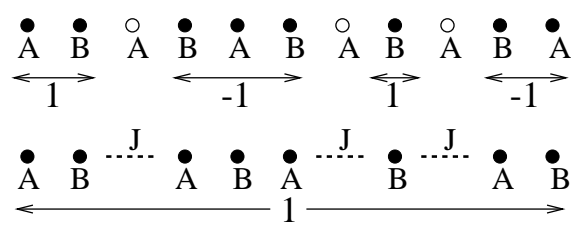

Fig. 1 - The emergence of the $Z_{2}$ "sublattice parity field" in the geometrical "squeezing" operation. The open circles refer to some configuration of holes. The black dots define the embedding space of the spin system in both the full (upper) and squeezed (lower) lattice. The unsqueezing operation can be parametrized in terms of binding of the electric charge quantum to flips in the $Z_{2}$-valued sublattice parity field.

by Woynarovich [6] and Ogata and Shiba [7], we construct a non-local operator measuring directly the topological order, which is a close relative of the string correlator of den Nijs and Rommelse [2]. Using the density matrix renormalization group method, we demonstrate numerically that this order is present in the Hubbard chain for all positive $U$ 's and electron densities. We subsequently show that a straightforward application of bosonization leads to difficulties in finding unambiguous inconsistencies, demonstrating that this topological order is not present in the Dirac vacuum in $1+1 \mathrm{D}$. Our non-local correlator has the structure of the Wilson loop of a $Z_{2}$ gauge theory. We will see that it may be possible to realize this state in a specific kind of $1+1 \mathrm{D}$ superconductor.

Woynarovich-Ogata-Shiba $[6,7]$ amounts to the demonstration that in the limit $U \rightarrow \infty$ the Bethe-Ansatz solution of the 1D Hubbard acquires a simple but peculiar structure. The wave function factorizes into a charge part $\psi_{\mathrm{SF}}$, depending on the electron coordinates $x_{i}$, and a spin part $\psi_{\mathrm{H}}$ which merely depends on the way the spins are distributed,

$$
\psi\left(\left\{x_{i}\right\}_{i=1}^{N} ;\left\{y_{j}\right\}_{j=1}^{N / 2}\right)=\psi_{\mathrm{SF}}\left(\left\{x_{i}\right\}_{i=1}^{N}\right) \psi_{H}\left(\left\{y_{j}\right\}_{j=1}^{N / 2}\right) .
$$

The charge part $\psi_{\mathrm{SF}}$ is the wave function of a system of non-interacting spinless fermions with coordinates $x_{i}$. The spin part $\psi_{\mathrm{H}}$ is identical to the wave function of a chain of Heisenberg spins interacting via a nearest-neighbor antiferromagnetic exchange. However, the positions $y_{i}$ of the up spins are needed but these no longer refer to the Hubbard chain. Instead, these parametrize another lattice with sites at coordinates $x_{1}, x_{2}, \ldots, x_{N}$ given by the positions of the charges in a configuration with amplitude $\psi_{\mathrm{SF}}$. Peculiarly, the quantum dynamics of interacting electrons has generated a geometrical structure which can be visualized by a representative example (fig. 1). Consider $N$ electrons on a chain with $L$ sites under the condition that $N<L$ such that the charge configurations can be specified by the locations of the holes. A charge configuration in the full Hubbard chain ("external space") has an amplitude $\psi_{\mathrm{SF}}$ in the wave function with the coordinates of the dots corresponding with the $x_{i}$ 's. The spin system sees a different "internal space" obtained from the full space by removing the holes together with the sites where the holes are located, substituting the hole and its site with an antiferromagnetic exchange between the sites neighboring the hole (the "squeezed space" [7]).

This is a very simple geometrical structure which can be formulated in terms of a simple topological "gauge" theory. In what aspects are the full chain and the squeezed chain different? The squeezed chain is obviously shorter than the full chain and this is a simple dilation: a distance $x$ measured in the full chain becomes a distance $\rho x$ in the squeezed chain $(\rho=N / L$, the electron density) when $x \gg 1$, the lattice constant. The other aspect is also simple, but less trivial in its consequences. The spin system is a quantum-antiferromagnet and is therefore sensitive to the geometrical property of bipartiteness. A lattice is called bipartite 
if it can be subdivided into two sublattices $A$ and $B$, such that all sites on the $A$ sublattice are neighbored by $B$ sublattice sites and vice versa. This division can be done in two ways $(\cdots-A-B-A-B \cdots$ and $\cdots-B-A-B-A \cdots)$ defining a $Z_{2}$ valued quantity "sublattice parity", $p= \pm 1$. Consider now what happens with sublattice parity in the squeezing. For the Heisenberg spin chain a redefinition of $p=1 \leftrightarrow-1$ does not carry any consequence ("pure gauge"). However, sublattice parity becomes important in the mapping of squeezed space into full space (fig. 1). "Fix the gauge" in squeezed space by choosing a particular sublattice parity, and consider what happens when it is unsqueezed. The holes are inserted, and because every hole is attached to one site, every time a hole is passed the sublattice parity flips. This is true for every instantaneous charge configuration, but the ground state is a superposition of many of these configurations: the charge is delocalized and it cannot be said where the sublattice flips are, but it is still certain that the sublattice parity will flip when a hole is passed. This is a form of topological order of a kind similar to that encountered in spin-1 chains. In the spin chain the sublattice parity flips are attached to the magnetic quantum number $M_{s}=0$ states and after squeezing these out a bipartite lattice results carrying a system of antiferromagnetically coupled Ising spins (corresponding with the $M_{s}= \pm 1$ states) which orders subsequently [2]. The topological order of the Luttinger liquid is richer, because the spin order parameter is superimposed on an additional charge degree of freedom which is missing in the spin-1 chains.

Can a correlation function be defined acting on the full Hubbard chain which can measure the true spin correlations living in squeezed space? Since all that matters is sublattice parity, this can be achieved by simply multiplying the spin operator by a factor -1 every time a hole is passed, thereby removing the sublattice parity flips from the spin correlations. Define staggered magnetization as $\vec{M}\left(x_{i}\right)=(-1)^{x_{i}} \vec{S}\left(x_{i}\right)$, where $\vec{S}$ is the spin operator $\left(S^{z}=\frac{1}{2}\left(n_{\uparrow}-\right.\right.$ $\left.\left.n_{\downarrow}\right), S^{+}=c_{\uparrow}^{\dagger} c_{\downarrow}\right)$ with the charge operator $n_{x_{i}}=n_{x_{i} \uparrow}+n_{x_{i} \downarrow}$ taking the values 0,1 and 2 for an empty, singly and doubly occupied site, respectively. The correlation function we are looking for is $[8]$

$$
O_{\text {top }}(x)=\left\langle M^{z}(0)(-1)^{\sum_{j=1}^{x-1} 1-n_{j}} M^{z}(x)\right\rangle .
$$

The operator $(-1)^{\left(1-n_{j}\right)}$ takes the value +1 for a singly occupied site, while it is -1 for a charge (hole, or doubly occupied) site. By multiplying these values on the interval $0<j<x$, all the minus signs associated with the sublattice parity flips are removed from the spin correlations.

The "string" [2] operator $(-1)^{\sum_{j}{ }^{1-n_{j}}}$ can be evaluated in a straightforward manner. Using the techniques by Parola and Sorella [9] for the large- $U$ limit,

$$
\left\langle M^{z}(0)(-1)^{\sum_{j=1}^{x-1} 1-n_{j}} M^{z}(x)\right\rangle=\sum_{j=2}^{x+1} P_{\mathrm{SF}}^{x}(j)(-1)^{j} O_{\mathrm{H}}(j-1),
$$

where $O_{\mathrm{H}}$ is the spin correlator of the Heisenberg chain, while $P_{\mathrm{SF}}^{x}(j)=\left\langle n(0) n(x) \delta\left(\sum_{l=0}^{x} n_{l}-\right.\right.$ $j)\rangle_{\mathrm{SF}}$ is the probability of finding $j$ spinless fermions in the interval $[0, x]$. This factor causes the additional decay of the spin correlations due to the charge fluctuations in the standard spin correlator. However, it is precisely compensated in $O_{\text {top }}$ by the factor $(-1)^{j}$ coming from the string operator and in the scaling limit we find

$$
O_{\text {top }}(x)=\frac{\rho}{x} \ln ^{1 / 2}(\rho x) .
$$

This is identical to the result for the pure Heisenberg spin chain $\frac{1}{x} \ln ^{1 / 2}(x)$ after rescaling the amplitude of staggered spin $\vec{M} \rightarrow \frac{\vec{M}}{\rho}$ and the measure of length $x \rightarrow x / \rho$, where $\rho$ is 


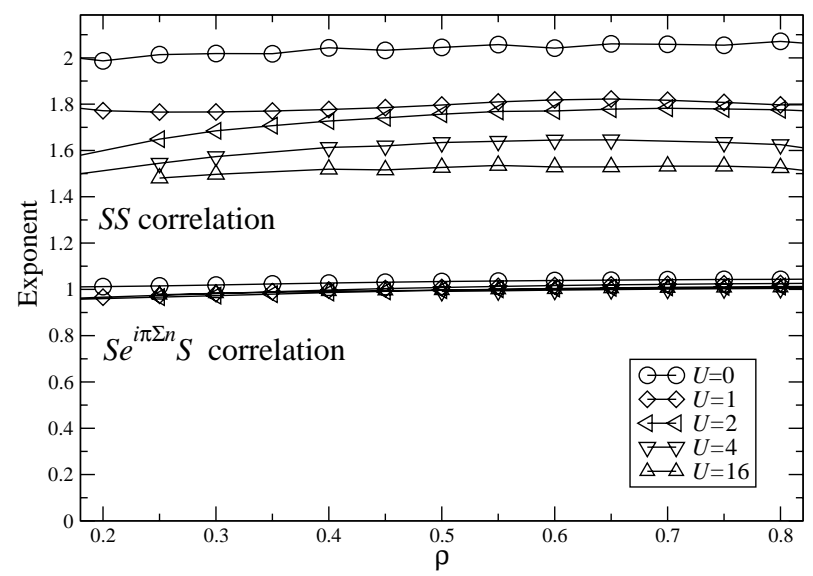

Fig. 2 - Exponents of the spin-spin correlation, $\eta$ (top) and the topological correlation, $\eta_{\text {top }}$ (bottom) as a function of electron filling, for various values of interaction $U$.

the average charge density. Comparing eq. (4) with the well-known [9] asymptotic behavior of the local staggered spin correlations for the Hubbard model, $O_{\vec{M}}(x)=\left\langle\left|M^{z}(0) M^{z}(x)\right|\right\rangle \sim$ $\frac{\cos \left(\left[2 k_{\mathrm{F}}-\pi\right] x\right)}{x^{1+K_{\rho}}}$, we find that the former decays more slowly. The charge stiffness $K_{\rho}$ is associated with the decay of the charge correlations, $\langle n(0) n(x)\rangle \sim \cos \left(4 k_{\mathrm{F}} x\right) / x^{4 K_{\rho}}$, and $K_{\rho}>0$ for all $\rho \neq 1$. Hence, the charge fluctuations modify the spin correlations because the charge is attached to the sublattice parity flips, resulting in a simple multiplicative factor $1 / x^{K_{\rho}}$. It follows that the comparison between the spin-spin correlator and eq. (2) yields a direct measure of the presence and strength of the topological order.

Is this topological order an accident of the strongly coupled case? Having identified the procedure to measure the topological order, it becomes possible to study it in any one-dimensional system. For finite $U$ it becomes very hard to calculate eq. (2) from the Bethe-Ansatz solution. The infinite $U$ point has a status similar to that of the special (AKLT) point in the spin chain where the hidden order becomes exact [2], and for finite $U$ the non-factorizability of the Bethe-Ansatz solution reflects the presence of short-distance fluctuations. We therefore compute it numerically for the Hubbard model using DMRG. We employ an algorithm recently developed by one of us [10], making explicit use of the $S U(2) \times S U(2)$ symmetry of the Hubbard model. In this formulation, the generators of the global symmetry group are the spin $\vec{S}$ and the pseudospin $\vec{I}$; the latter is a generalization from the charge $U(1)$ to an $S U(2)$ symmetry such that the particle number at site $i$ is given by $n_{i}=1+2 I_{i}^{z}$. In principle, it is straightforward to calculate the expectation value of string operators such as eq. (2) using the DMRG method. However, the calculation of critical correlators with DMRG is not unproblematic even in $1+1 \mathrm{D}$, because of the truncation errors causing eventually an exponential decay [11], and the requirement to use open boundaries. We used a relatively large system size (1000 sites) to reduce the effect of the boundary conditions, and used a basis size (700 $S U(2) \times S U(2)$ states) large enough to achieve a truncation length of the order of 200-300 lattice constants. In this way meaningful results can be obtained from a simple curve fit to obtain the desired exponents.

In fig. 2 we show our results for the exponents of both the standard two-point spin correlator $\langle\vec{M} \cdot(0) \vec{M}(x)\rangle$, and our topological correlator, eq. (2), as a function of density for various interaction strengths. The former illustrates the quality of the calculation; the dominant 
correlation is at the wave vector $2 k_{\mathrm{F}}-\pi, \sim \cos \left(\left[2 k_{\mathrm{F}}-\pi\right] x\right) / x^{\eta}$ and it is seen from fig. 2 that the exponent $\eta$ depends strongly on the parameters. In fact, the exponent behaves exactly according to the expectations: $\eta=K_{\sigma}+K_{\rho}$, where $K_{\sigma}=1$ and $K_{\rho}$ is consistent with the values previously obtained by Schulz [12]. Considering now the topological correlator, the dominant component lives at the wave vector $q=0$; we find no other characteristic momenta in the Fourier transform of eq. (2) which is already reminiscent of the staggered spin correlator of a Heisenberg chain. This is fortified by our finding that the exponent $\eta_{\text {top }}$ does not depend on the microscopic parameters at all. In fact, for all parameters the exponent of the topological correlator $\eta_{\text {top }}=1=K_{\sigma}$. Hence, regardless of the values of $U$ and $\rho$ the long-distance behavior of the topological correlator is indistinguishable from the spin-spin correlator of a Heisenberg chain, demonstrating that the Hubbard model indeed carries the sublattice parity topological order fully for all values $U>0$.

If the topological order were complete, the amplitude of $O_{\text {top }}$ would be identical to that of the Heisenberg model, after rescaling the spin amplitude and length measure. This is realized in the $U \rightarrow \infty$ limit, but for finite coupling the factorization property of the wave function is destroyed by local fluctuations and this is reflected in a smaller (but still non-zero) amplitude of $O_{\text {top }}$ for small $U$.

Let us now explain the reasons why such a topological order cannot be present in the true Dirac vacuum. At the center of our considerations for the lattice model is the fact that the topological structure is $Z_{2}$-valued (the sublattice parity) and this in turns rests on the fact that on a lattice the number operator $n_{i}$ is integer-valued: a site is either empty, singly or doubly occupied and one isolates the evenness or unevenness by exponentiation, $\sigma_{i}^{3}=(-1)^{n_{i}}= \pm 1$. In a relativistic theory, one has to keep the fermion creation and annihilation operator apart, $n_{r} \rightarrow \psi^{\dagger}(r) \psi(r+\epsilon)$, and the resulting Schwinger terms are the building blocks for bosonization. Thereby, the charge density becomes the gradient of a continuous function $n(x)=(1 / \pi) \partial_{x}$. Accordingly, $(-1)^{n_{i}} \sim(-1)^{\partial_{x} \phi}$ is a complex scalar and not an Ising-valued operator. This reflects the fact that it is impossible to localize an electron in space in a truly relativistic theory (UV cut-off at infinity), while the ability to ascribe a position to the electron is at the heart of the topological order on the lattice.

In fact, this problem is to a certain extent repairable. The operator entering the string correlator is not $\sigma^{3}$ itself, but instead $\Pi_{l} \sigma^{3}=\exp \left[i \pi \int_{x}^{x^{\prime}} \mathrm{d} x n(x)\right]$, taking the continuum limit. The integral smooths out the discreteness, and an integral over the continuous bosonization fields can be manipulated to mimic the product of Ising-valued operators. This is a subtle UV regularization issue, which we will discuss elsewhere in detail [13]. To give the reader some feeling, let us consider the expectation value of the string correlator itself,

$$
\left\langle(-1)^{\sum_{j=0}^{x} n_{j}}\right\rangle=\left\langle\cos \left[\pi \sum_{j} n(j)\right]\right\rangle \longrightarrow\left\langle\cos \left[\pi \int_{0}^{x} \mathrm{~d} y n(y)\right]\right\rangle .
$$

Accordingly, the total charge density is $n(x)=\sqrt{\frac{2}{\pi}} \frac{\partial \varphi_{c}}{\partial x}(j)+\mathcal{O}_{\mathrm{CDW}}(j)+\mathcal{O}^{\dagger} \mathrm{CDW}(j)$, where $\mathcal{O}_{\mathrm{CDW}}(x)=\exp \left[-2 i k_{\mathrm{F}} x\right] \exp \left[i \sqrt{2 \pi} \varphi_{c}\right]=\cos \left(\sqrt{2 \pi} \varphi_{s}\right)$. It follows that

$$
\left\langle(-1)^{\sum_{j=0}^{x} n_{j}}\right\rangle=\left\langle\exp \left[i \sqrt{2 \pi}\left[\varphi_{c}(x)-\varphi_{c}(0)\right]\right]\right\rangle=\cos \left(2 k_{\mathrm{F}} x\right) / x^{K_{\rho}} .
$$

On the other hand, because on the lattice $(-1)^{n_{i}} \equiv(-1)^{2 S_{i}^{z}}$ we might as well use

$$
\left\langle(-1)^{\sum_{j=0}^{x} n_{t o t}(j)}\right\rangle=\left\langle\cos \left[2 \pi \sum_{j=0}^{x} S^{z}(j)\right]\right\rangle \text {. }
$$


Using the bosonized form $2 S^{z}(x)=\sqrt{\frac{2}{\pi}} \frac{\partial \varphi_{s}}{\partial x}(j)+\mathcal{O}_{\mathrm{SDW}}(j)+\mathcal{O}_{\mathrm{SDW}}^{\dagger}(j)$ it follows that

$$
\left\langle(-1)^{\sum_{j=0}^{x} n_{j}}\right\rangle=\frac{1}{x^{K_{\sigma}}}
$$

For $U=0, K_{\sigma}=K_{\rho}$ and the two expressions are the same, but away from this point $K_{\sigma} \neq K_{\rho}$ and depending the way one calculates $D$, one obtains different answers. On the other hand, the numerical calculations show that the correct expression is

$$
\left\langle(-1)^{\sum_{j=0}^{x} n_{j}}\right\rangle=A / x^{\theta}+B \cos \left(2 k_{\mathrm{F}} x\right) / x^{K_{\rho}},
$$

where $A$ and $B$ are non-universal amplitudes [14]. The exponent $\theta$ is difficult to pin down as the corresponding amplitude vanishes exponentially for $U>0$, however it is clearly related to the presence of double occupied sites in the charge distribution, possibly $\theta=1 / K_{\rho}$. Indeed, this operator cannot measure $K_{\sigma}$ because it is transparent to the direction of spin. Bosonization relies on point splitting to be able to form a continuum limit, and therefore effects arising from double occupied sites on a lattice are not properly taken into account. In the presence of point splitting $(-1)^{n_{i}} \neq(-1)^{2 S_{i}^{z}}$ and more care is required to recover the correct bosonized expression. It should be emphasized that this is additional to the well-understood lack of knowledge of the $k=0$ component of the density, which gives corrections of order $\cos \left(2 k_{\mathrm{F}}\right)$.

In summary, we have discovered a correlation function which makes it possible to measure directly the presence of a hidden or topological order underlying spin-charge separation in $1+1 \mathrm{D}$ electron systems. This hidden order can be viewed as a geometrical structure where the spin system lives in a squeezed space different from the full chain, and it is related but different to the hidden order in the $S=1$ spin chains. The suspicion was widespread that the Woynarovich-Ogata-Shiba construction was special to the $U \rightarrow \infty$ limit and our main result is the numerical demonstration that instead the hidden order is generic in the scaling limit. This insight is powerful enough to hint at the existence of hitherto unidentified states of $1+1 \mathrm{D}$ matter. An exciting possibility is closely related to recent ideas regarding a potential connection between Ising gauge theory and the destruction of stripe order in $2+1$ dimensions $[4,15]$. As we discussed, $(-1)^{n_{i}}$ is Ising-valued and the string operator can be written accordingly as $\Pi_{i} \sigma_{i}^{3}$, where $\sigma_{i}^{3}$ represents the flipping of the sublattice parity itself. A local gauge transformation preserves the property that $\Pi_{i} \sigma_{i}^{3}=(-1)^{\sum_{i} n_{i}}$, hence the correlator $O_{\text {top }}(x)$ is equivalent to a Wilson line of $Z_{2}$ gauge theory [16]. Hence, if local Ising symmetry were dictating, $\left\langle S \Pi \sigma^{3} S\right\rangle \sim \exp \left[x / \xi_{\mathrm{g}}\right]\langle S S\rangle$, because the l.h.s. is gauge invariant while the r.h.s. is not; $\xi_{\mathrm{g}}$ is the length scale where the gauge invariance emerges. In the Luttinger liquid the two are related by an algebraic factor $1 / x^{K_{\rho}}$ but this is due to the algebraic order in the charge system. Therefore, under the conditions that a) the spin system stays separated from the charge, b) the charge quanta are bound to sublattice parity flips, and c) the charge correlations become short range, one will obtain the required relations between the correlation functions signaling the $Z_{2}$ gauge invariance. Condition c) is generally satisfied when the superconducting phase is forced to condense by applying an external Josephson field. The attractive $U$ Hubbard model fails in this regard because its ground state can be viewed as a continuation of the local pair limit where the spin system is destroyed.

However, it is easy to convince oneself that the above criteria are precisely fulfilled by the Haldane spin chain, becoming particularly obvious in the Batista-Ortiz interpretation [3]. Therefore, one way to characterize the symmetry difference between the spin-chain and the Luttinger liquid is that the spin-chain is characterized by a local $Z_{2}$ invariance and the Luttinger liquid is not. However, the den Nijs-Rommelse case, where the topological correlator 
shows true long-range order, is special in that the gauge fluctuations are completely suppressed, i.e. the gauge coupling goes to infinity. If the $S U(2)$ spin symmetry of the fermionic model is restored (thus eliminating the spin gap while maintaining the charge condensate), we expect the topological correlator to decay exponentially more slowly than the spin-spin correlator but this time with algebraic decay. These conditions might well be satisfied in $t-J$ ladders under the influence of an external Josephson field [17].

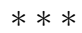

We acknowledge stimulating discussions with M. Gulácsi, G. Ortiz, S. A. Kivelson, S. Sachdev, F. Wilczek, T. Becker, E. Fradkin, N. Nagaosa and T. K. Ng. Financial support was provided by the Foundation of Fundamental Research on Matter (FOM), which is sponsored by the Netherlands Organization for the Advancement of Pure Research (NWO). Numerical calculations were performed at the National Facility of the Australian Partnership for Advanced Computation, through the Supercomputer Time Allocation Committee at the Australian National University.

\section{REFERENCES}

[1] Voit J., Rep. Prog. Phys., 58 (1995) 977.

[2] Den Nijs M. P. M. and Rommelse K. J., Phys. Rev. B, 40 (1989) 4709.

[3] Batista C. D. and Ortiz G., Phys. Rev. Lett., 86 (2001) 1082.

[4] Zaanen J., Osman O. Y., Kruis H. V., Nussinov Z. and Tworzydlo J., Philos. Mag. B, 81 (2002) 1985; Nussinov Z. and ZaAnen J., cond-mat/0209437; ZAanen J. and Nussinov Z., cond-mat/0209441.

[5] Lieb E. H. and Wu F. Y., Phys. Rev. Lett., 20 (1968) 1445.

[6] Woynarovich F., J. Phys. C, 15 (1982) 85; 96.

[7] Ogata M. and Shiba H., Phys. Rev. B, 41 (1990) 2326.

[8] This correlator was introduced first in the context of the dynamical stripes: see ZAANEN J. and van SaArloos W., Physica $C, \mathbf{2 8 2}$ (1997) 178. Similar constructions were used by Weng Z. Y., Phys. Rev. B, 50 (1994) 13837 to address phase shifts in fermion propagators in the large- $U$ limit.

[9] Parola A. and Sorella S., Phys. Rev. Lett., 64 (1990) 1831.

[10] McCulloch I. P. and Gulácsi M., Europhys. Lett., 57 (2002) 852.

[11] Östlund S. and Rommer S., Phys. Rev. Lett., 75 (1995) 3537.

[12] Schulz H. J., Phys. Rev. Lett., 64 (1990) 2831.

[13] Kruis H. V., McCulloch I. P., Nussinov Z. and ZaAnen J., in preparation.

[14] Despite claims to the contrary (Strong S. P. and Talstra J. C., Phys. Rev. B, 59 (1999) 7362; Nakamura M. and Voit J., Phys. Rev. B, 65 (2002) 153110), it follows from our results that this correlator is not an appropriate measure of hidden order.

[15] Zhang Y., Demler E. and Sachdev S., Phys. Rev. B, 66 (2002) 094501; Sachdev S. and Morinari T., cond-mat/0207167; SACHDEV S., cond-mat/0211027.

[16] Kogut J. B., Rev. Mod. Phys., 51 (1979) 659.

[17] White S. R. and Scalapino D. J., Phys. Rev. Lett., 81 (1998) 3227. 